\title{
LA DISPONIBILIDAD LÉXICA EN ESPAÑOL DE ALUMNOS MARROQUÍES DE ENSEÑANZA MEDIA: RESULTADOS GENERALES
}

\author{
Lahoussine Aabidi \\ Universidad de Las Palmas de Gran Canaria
}

\begin{abstract}
RESUMEN: Esta investigación forma parte de los estudios de léxico encargados de analizar el vocabulario disponible en español de los aprendientes extranjeros mediante el recuento y examen del vocabulario recopilado. El estudio de léxico disponible en ELE de alumnos marroquíes de enseñanza secundaria se basa principalmente en los resultados que ofrecen los tres parámetros estadísticos que se suelen tomar en cuenta: la producción de palabras, la producción de vocablos y el índice de cohesión. Compararemos también nuestros resultados cuantitativos con los de otras investigaciones de vocabulario disponible en español como lengua materna y extranjera.
\end{abstract}

PALABRAS CLAVE: léxico disponible, español como lengua extranjera (ELE), enseñanza secundaria, vocabulario, Proyecto Panhispánico de Disponibilidad Léxica

Lexical availability in Spanish of Moroccan secondary school students: general results

\begin{abstract}
This paper is part of the studies that employ quantitative methods to examine the available vocabulary in Spanish of foreign language learners. The analysis of the lexical availability in SFL of Moroccan high school students is based on the results offered by the three parameters that are usually taken into account in these studies: the production of answers, the production of different words, and the cohesion index. We will also compare our quantitative results with those of other works that analyze the available vocabulary in Spanish as either a native or a foreign language.
\end{abstract}

KEYWORDS: available lexicon, Spanish as a foreign language (SFL), secondary education, vocabulary, Pan-Hispanic Project of Lexical Availability

\section{INTRODUCCIÓN}

Esta investigación forma parte de los estudios de léxico encargados de analizar el vocabulario disponible en español de los aprendientes extranjeros. La aplicación de los principios metodológicos del Proyecto Panhispánico de Disponibilidad Léxica (PPHDL) al español como lengua extranjera acumula ya más de dos décadas de investigación desde que, a finales de los años noventa del siglo pasado, Carcedo González (1997-1998) 
estudiara el léxico disponible en español de los alumnos finlandeses. ${ }^{1}$ Posteriormente, Samper Hernández (2002) emplea dicha metodología para analizar el vocabulario disponible en español de estudiantes extranjeros que se encuentran en situación de inmersión lingüística. Estos dos investigadores son quienes han sentado las bases de esta línea científica, cuyo objetivo principal es recopilar y analizar el vocabulario español de los aprendientes extranjeros con el fin de buscar nuevas propuestas y vías metodológicas capaces de mejorar el material didáctico de ELE y garantizar un buen aprendizaje de este idioma.

En Marruecos el idioma español ha tenido una situación precaria en el sistema educativo nacional, sobre todo después de la descolonización, cuando las autoridades marroquíes adoptaron el plan de marroquinización y la implantación del francés como segunda lengua oficial después del árabe (Benyaya, 2007). Hoy en día, a pesar del esfuerzo durante muchas décadas de España por implantar su lengua y su cultura en el sistema educativo marroquí, el español suele ocupar el tercer lugar después del francés y del inglés. Además, mucha parte del patrimonio cultural español va desapareciendo, incluso en las zonas que constituyeron las excolonias españolas (Charia, 2014).

El objetivo del presente estudio es analizar el léxico disponible en español como lengua extranjera de los alumnos marroquíes de enseñanza media. Se trata principalmente de exponer los resultados generales desde el punto de vista cuantitativo y de comparar estos resultados con los de otras investigaciones de vocabulario disponible en español como lengua materna y extranjera, que emplean también la metodología del Proyecto Panhispánico de Disponibilidad Léxica. ${ }^{2}$ Para alcanzar este objetivo, nos basaremos en el análisis de los resultados que ofrecen los tres parámetros estadísticos que se suelen emplear en los estudios de esta misma índole (vid. López González, 2014), y que forman parte del Proyecto Panhispánico. Dichos criterios cuantitativos son la producción de palabras, la producción de vocablos y el índice de cohesión. El propósito final es mejorar la enseñanza del español a alumnos marroquíes, sobre todo en lo referente a la selección y planificación del vocabulario que debe usarse en los manuales escolares.

\section{Aspectos METODOLÓGICOS}

En la realización de este trabajo se aplican las diferentes pautas metodológicas que rigen las investigaciones de vocabulario disponible dentro del PPHDL. Es una metodología rigurosa que ha acumulado varias décadas de experimentación científica desde la aparición de este proyecto a finales del siglo pasado. La edición de los materiales se basará principalmente en las normas comunes propuestas por Samper Padilla (1998) y en los criterios específicos planteados por Samper Hernández (2002, 27-30).

Para recoger el corpus objeto de estudio de este trabajo, se han utilizado unas encuestas abiertas que han sido rellenadas en 16 institutos de enseñanza media marroquí 
de las Academias Regionales de Educación y Formación de Sus-Massa, El Aaiún-Saguia El Hamra y Dakhla-Oued Eddahab. ${ }^{3}$ Las encuestas se componen de dos partes. En la primera se deben introducir informaciones sociolingüísticas del alumno (datos personales y académicos, nivel educativo y profesión de los padres y ubicación del centro escolar). Son datos que permiten la estratificación posterior de los encuestados según las variables que se quieren contemplar. En la segunda parte figura a la cabeza de cada página el título de tres centros de interés, seguidos de unas líneas trazadas donde el informante debe escribir todas las palabras que le sugiere cada estímulo verbal en un límite temporal de dos minutos por cada centro.

Dichos centros de interés son los mismos que se han empleado en las investigaciones pioneras, y que se supone que son capaces de actualizar el caudal léxico relacionado con los diferentes ámbitos de la vida cotidiana. Se trata de los campos asociativos 01. "Partes del cuerpo" (CUE), 02. "La ropa” (ROP), 03. "Partes de la casa (sin los muebles)" (CAS), 04. "Los muebles de la casa" (MUE), 05. "Alimentos y bebidas" (ALI), o6. "Objetos colocados en la mesa para la comida" (MES), 07. "La cocina y sus utensilios" (COC), 08. "La escuela: muebles y materiales" (ESC), 09. "Iluminación, calefacción y medios de airear un recinto" (ILU), 10. "La ciudad" (CIU), 11. "El campo" (CAM), 12. "Medios de transporte" (TRA), 13. "Trabajos del campo y del jardín” (TRC), 14. "Los animales" (ANI), 15. "Juegos y distracciones" (JUE), 16. "Profesiones y oficios" (PRO) y 17. "Los colores" (COL). Se ha añadido también este último ámbito temático ('Los colores'), puesto que viene siendo una constante, sobre todo en las investigaciones de léxico disponible en español como lengua extranjera (Serfati y Aabidi, 2013; Jiménez Berrio, 2013; Sandu, 2014; Gallego Gallego, 2014; Mendoza Puertas, 2018).

En el sistema educativo marroquí, el aprendizaje de una segunda lengua extranjera -el español o el inglés- es obligatorio a partir del último curso de la enseñanza colegial (14 y 15 años) y en los tres cursos de la enseñanza secundaria cualificante (15 a 18 años). En la muestra objeto de este análisis, nuestros informantes son adolescentes que aprenden ELE en los tres niveles de la enseñanza media: inicial (90 alumnos cuya edad oscila entre 15 y 16 años), intermedio (120 encuestados de entre 16 y 17 años) y avanzado (140 informantes de unos 18 años), que corresponden, aproximadamente, a los niveles de referencia A1, A2 y B1 establecidos en el Plan curricular del Instituto Cervantes (2007). Son en total 350 encuestados, un número que no está lejos del tamaño de las muestras (40o sujetos) acordado por los especialistas en disponibilidad léxica en la reunión de Bilbao (1999). Estos informantes representan casi el $9 \%$ del total de alumnos que estudian ELE en las Academias Regionales de Educación y Formación objeto de este estudio. Los encuestados han sido estratificados, por otra parte, según cinco factores sociolingüísticos: sexo, lengua materna, zona geográfica, nivel escolar y nivel sociocultural. Destaca la desigualdad en el número de sujetos que forman las variantes de cada variable, puesto que no hubo una estratificación predeterminada de la muestra. En 
la tabla 1 se recogen tanto los factores sociolingüísticos como sus variantes y la proporción que representa cada una.

\begin{tabular}{|c|c|c|c|c|c|}
\hline & Sexo & $\begin{array}{l}\text { Lengua } \\
\text { materna }\end{array}$ & Zona geográfica & Nivel escolar & $\begin{array}{c}\text { Nivel } \\
\text { sociocultural } 4\end{array}$ \\
\hline Masculino & $\begin{array}{l}156 \\
(44.57 \%)\end{array}$ & & & & \\
\hline Femenino & $\begin{array}{l}194 \\
(55.42 \%)\end{array}$ & & & & \\
\hline Árabe & & $236(67.42 \%)$ & & & \\
\hline Bereber & & $114(32.57 \%)$ & & & \\
\hline Excolonia francesa & & & $125(35.71 \%)$ & & \\
\hline Excolonia española & & & $225(64.28 \%)$ & & \\
\hline Tronco común (A1) & & & & $90(25.71 \%)$ & \\
\hline $1^{\circ}$ Bachillerato (A2) & & & & $120(34.28 \%)$ & \\
\hline $2^{\circ}$ Bachillerato (B1) & & & & $140(40 \%)$ & \\
\hline Bajo & & & & & $188(53.71 \%)$ \\
\hline Medio bajo & & & & & $140(40 \%)$ \\
\hline Medio & & & & & $17(4.85 \%)$ \\
\hline Medio alto & & & & & $5(1.42 \%)$ \\
\hline
\end{tabular}

Tabla 1. Distribución de la muestra según las variables sociolingüísticas contempladas

Después de recopilar el material necesario para llevar a cabo este estudio, hemos procedido a la edición del vocabulario producido por los encuestados de acuerdo a las normas homogéneas del PPHDL, tales como la corrección ortográfica, la eliminación de las unidades repetidas en un mismo centro, la neutralización de variantes meramente flexivas y la unificación de los derivados que no suponen alteración del significado (Samper Padilla, 1998). Además de estas normas, hemos aplicado también los criterios de edición específicos que suelen emplear los investigadores de vocabulario disponible en ELE (Samper Hernández, 2002, 27- 30).

Tras la limpieza del material recogido y su preparación bajo forma de ficheros Word, llega la última fase metodológica que permite la obtención de los resultados. Se trata de la introducción de dichos ficheros en el programa informático LexiDisp, con el fin de conseguir los índices estadísticos necesarios.

\section{RESULTADOS GENERALES: ANÁLISIS Y COMENTARIO}

El análisis de los resultados generales obtenidos tras el cálculo de la disponibilidad léxica de los marroquíes que aprenden español en la enseñanza media permite conocer el número tanto de respuestas como de vocablos producidos a partir de los estímulos verbales contemplados. Dichos resultados ofrecen también un diagnóstico acerca de la 
productividad y diversificación léxicas de los sujetos encuestados. A continuación, se analizan estos resultados proporcionados por los diferentes parámetros cuantitativos que se suelen emplear en los estudios de vocabulario disponible dentro del PPHDL.

\section{1. Producción de palabras}

La cantidad de palabras que han aportado los alumnos marroquíes de ELE en este estudio alcanza un total de 39462 respuestas, número que se puede traducir en una media de 2321.35 unidades léxicas por ámbito temático y de 112.75 por informante. En la tabla 2 se presentan los totales de respuestas producidas en cada área temática, así como la posición que ocupa cada centro y el promedio de palabras por sujeto. Lo primero que llama la atención es la gran distancia numérica registrada entre el campo nocional o8. 'ESC' y el ámbito 06. 'MES', que ocupan la primera y la última posición, respectivamente: 3490 palabras frente a 1366 unidades.

\begin{tabular}{|l|c|c|c|}
\hline CI & Rango & Palabras & Promedio por sujeto \\
\hline 01. CUE & 05 & 2711 & 7.7 \\
\hline 02. ROP & 13 & 1756 & 6.6 \\
\hline 03. CAS & 07 & 2319 & 7.2 \\
\hline 04. MUE & 06 & 2547 & 9.1 \\
\hline 05. ALI & 03 & 3191 & 3.9 \\
\hline 06. MES & 17 & 1366 & 5.7 \\
\hline 07. COC & 12 & 2014 & 9.9 \\
\hline 08. ESC & 01 & 3490 & 4.6 \\
\hline 09. ILU & 15 & 1640 & 9.7 \\
\hline 10. CIU & 02 & 3428 & 5.9 \\
\hline 11. CAM & 11 & 2081 & 6.5 \\
\hline 12. TRA & 09 & 2308 & 4.5 \\
\hline 13. TRC & 16 & 1589 & 4.9 \\
\hline 14. ANI & 14 & 1739 & 6.6 \\
\hline 15. JUE & 07 & 2319 & 8.2 \\
\hline 16. PRO & 04 & 2882 & 5.9 \\
\hline 17. COL & 10 & 2082 & 6.58 \\
\hline Total & -- & 39462 & \\
\hline
\end{tabular}

Tabla 2. Palabras por centro de interés

Los resultados expuestos en esta tabla muestran que los centros de interés más productivos según el número de palabras son el o8. 'ESC', el 10. 'CIU' y el o5. 'ALI', puesto que registran el mayor número de respuestas y consiguen, por lo tanto, los promedios más altos: 9.9, 9.7 y 9.1 puntos, respectivamente. Se trata de unos ámbitos 
temáticos muy presentes en los manuales escolares de ELE en Marruecos y, además, son temas que hacen referencia a realidades muy cercanas a la vida cotidiana de los alumnos. Estos mismos ámbitos temáticos destacan generalmente como centros muy productivos en muchas investigaciones de léxico disponible en ELE. En el caso de los aprendientes rumanos (Sandu, 2014), por ejemplo, el centro 05. 'ALI' ocupa la primera posición, obteniendo el mayor promedio (14.77 puntos). También figura en este mismo puesto en los estudios realizados en Finlandia (Carcedo González, 1997-1998; 2000) y Polonia (López González, 2010), mientras que en el presente trabajo y en el de China (Lin, 2012) aparece en la tercera posición. El campo 10. 'CIU', por su parte, ocupa el segundo rango en los polacos, mientras que en los finlandeses y en los rumanos figura en el tercer lugar. Sin embargo, llama la atención que el o8. 'ESC', que despunta en nuestro estudio, no aparece en las tres primeras posiciones ni en esas investigaciones ni en las realizadas con los nativos dentro del PPHDL (Samper Padilla y Samper Hernández, 2006). Por ello, consideramos oportuno extender la lista de los campos más productivos y exponer, a continuación (tabla 3), los centros que ocupan los cinco primeros puestos en las investigaciones de léxico disponible en español, sea como lengua materna o extranjera, para descubrir qué similitudes y divergencias de rango se registran.

\begin{tabular}{|c|c|c|c|c|c|c|}
\hline Rango & Marruecos & Finlandia & China & Polonia & Rumanía & PPHDL $^{5}$ \\
\hline $1^{\circ}$ & 08. 'ESC' & 05. 'ALI' & 14. 'ANI' & 05. 'ALI' & 05. 'ALI' & 14. 'ANI' \\
\hline $2^{\circ}$ & 10. 'CIU' & 01. 'CUE' & 16. 'PRO' & 10. 'CIU' & 01. 'CUE' & 05. 'ALI' \\
\hline $3^{\circ}$ & 05. 'ALI' & 10. 'CIU' & 05. 'ALI' & 14. 'ANI' & 10. 'CIU' & "01. 'CUE' \\
\hline $4^{\circ}$ & 16. 'PRO' & 14. 'ANI' & 01. 'CUE' & 01. 'CUE' & 14. 'ANI' & 08. 'ESC' \\
\hline $5^{\circ}$ & 01. 'CUE' & 16. 'PRO' & 10. 'CIU' & 16. 'PRO' & 11. 'CAM' & 10. 'CIU' \\
\hline
\end{tabular}

Tabla 3. Comparativa de los cinco primeros centros más productivos

Como se puede observar, resulta llamativo que el centro más productivo (o8. 'ESC') en los marroquíes no aparece en la lista de los campos que ocupan los cinco primeros rangos en ninguna de las cuatro investigaciones de léxico disponible en ELE. Sin embargo, destaca su aparición en el cuarto lugar en los estudios del PPHDL. Por su parte, el centro 16. 'PRO', que ocupa la cuarta posición en nuestro trabajo, forma parte de los cinco primeros ámbitos más productivos en solo tres comunidades (Finlandia, China y Polonia), mientras que en las investigaciones tanto del PPHDL como de Rumanía figura en el séptimo puesto. Por otro lado, cabe señalar que tres de los campos asociativos más productivos en Marruecos (10. 'CIU', 05. 'ALI' y 01. 'CUE') aparecen también en la lista de los centros que ocupan los cinco primeros rangos en el resto de investigaciones. Por último, llama nuestra atención que el ámbito temático 14. 'ANI' figura como uno de los 
centros más productivos en los otros cinco estudios, mientras que en el caso de Marruecos ostenta una posición muy rezagada $\left(14^{\circ}\right)$.

Respecto a los ámbitos temáticos que se caracterizan por su menor producción léxica, nuestros alumnos han aportado pocas respuestas en 06. 'MES', 13. 'TRC' y 09. 'ILU'. La cantidad de unidades léxicas incluidas en estos centros no ha superado, por este orden, 1366, 1589 y 1640 respuestas, lo que corresponde a 3.9, 4.5 y 4.6 puntos de promedio, respectivamente. La pobreza léxica caracteriza también estos campos asociativos en los diferentes estudios de esta misma índole. El centro 13. 'TRC', a modo de ejemplo, ocupa la última posición en las investigaciones realizadas en China, Finlandia, Polonia y Rumanía. Casi pasa lo mismo con el centro 09. 'ILU' que figura en el penúltimo rango en los alumnos rumanos y chinos, mientras que en los finlandeses aparece en el antepenúltimo puesto. Estos tres ámbitos temáticos forman parte también de las áreas más pobres en los estudios de léxico dentro del PPHDL y suelen aparecer en posiciones muy rezagadas. Por otro lado, cabe subrayar que dos de los centros más pobres (O2. 'ROP' y 14. 'ANI') en el caso de Marruecos no aparecen en la lista de los centros menos productivos en los otros estudios. Por el contrario, y tal como señalamos anteriormente, el campo 14. 'ANI' se ha considerado como uno de los ámbitos más ricos en palabras en esas cinco investigaciones.

A continuación, en la tabla 4 se expone el rango de los cinco centros de interés menos productivos en los seis estudios cotejados.

\begin{tabular}{|c|l|l|l|l|l||l|}
\hline Rango & Marruecos & Finlandia & China & Polonia & Rumanía & PPHDL \\
\hline 12 ó 13 & 02. 'ROP' & 08. 'ESC' & 08. 'ESC' & 03. 'CAS' & 07. 'COC' & 03. 'CAS' \\
\hline 13 ó 14 & 14. 'ANI' & 06. 'MES' & 06. 'MES' & 07. 'COC' & 03. 'CAS' & 06. 'MES' \\
\hline Antepenúltimo & 09. 'ILU' & 09. 'ILU' & 07. 'COC' & 09. 'ILU' & 06. 'MES' & 04. 'MUE' \\
\hline Penúltimo & 13. 'TRC' & 07. 'COC' & 09. 'ILU' & 06. 'MES' & 09. 'ILU' & 09. 'ILU' \\
\hline Último & 06. 'MES' & 13. 'TRC' & 13. 'TRC' & 13. 'TRC' & 13. 'TRC' & 13. 'TRC' \\
\hline
\end{tabular}

Tabla 4. Comparativa de los cinco últimos centros menos productivos

La escasa producción léxica en algunas áreas temáticas invita a reflexionar sobre la utilidad de estos centros para recoger el verdadero vocabulario disponible de los alumnos en ELE y la necesidad de adaptarlos a las nociones específicas del Plan curricular del Instituto Cervantes. Es una observación que pone en tela de juicio también la eficacia de los métodos que se emplean para seleccionar y planificar el vocabulario propuesto para su explotación didáctica en las aulas.

Por otra parte, dicha pobreza léxica en estos centros de interés se manifiesta en casi todos los ámbitos geográficos (Samper Padilla, Bellón Fernández y Samper Hernández, 2003, 59), pero se puede ver acentuada en nuestro caso por razones de tipo sociocultural 
o económico. En el campo 06. 'MES', a modo de ejemplo, las familias marroquíes, sobre todo las que pertenecen a las clases sociales bajas no suelen poner muchos utensilios y platos sobre la mesa a la hora de comer, sino que tienen la costumbre de compartir generalmente un solo plato. Respecto a los otros dos ámbitos, es decir, el 13. 'TRC' y el o9. 'ILU', además de su escasa presencia en los métodos marroquíes de ELE, la mayoría de los encuestados vive en las ciudades y ya no tiene mucho contacto con las diferentes actividades agrícolas y ganaderas que realizan los campesinos. Estos alumnos tampoco suelen utilizar diferentes aparatos de climatización y calefacción, puesto que son en su mayoría informantes que pertenecen al estrato sociocultural bajo y medio-bajo (vid. tabla 1) y no disponen, por lo tanto, de esos aparatos en sus casas. Además, hay que subrayar que las condiciones climáticas del país no son muy duras, sobre todo en las regiones costeras del sur de Marruecos, y no permiten al alumnado, por consiguiente, que disponga de mucho vocabulario sobre el particular.

Para terminar este apartado, la producción de palabras aportadas en esta investigación permite diferenciar los campos asociativos en cuatro grupos de acuerdo a la clasificación de los mismos en los estudios de léxico disponible en español como lengua materna, realizada por Samper Padilla et al. (2003):7

\begin{tabular}{|c|c|c|}
\hline Grupo de centros & En los estudios del PPHDL & En el presente estudio \\
\hline $\begin{array}{l}1^{\text {er }} \text { grupo (centros más } \\
\text { productivos) }\end{array}$ & 01. 'CUE', 05. 'ALI', 14. 'ANI' & 05. 'ALI', 08. 'ESC', 10. 'CIU' \\
\hline $\begin{array}{l}2^{\circ} \text { grupo (campos que } \\
\text { obtienen o superan la media) }\end{array}$ & $\begin{array}{l}\text { 02. 'ROP', 8. 'ESC', 10. 'CIU', } \\
\text { 11. 'CAM', 16. 'PRO' }\end{array}$ & $\begin{array}{l}\text { 01. 'CUE', 03. 'CAS', 04. 'MUE', } \\
\text { 15. 'JUE', 16. 'PRO' }\end{array}$ \\
\hline $\begin{array}{l}3^{\text {er grupo (centros que no }} \\
\text { están lejos de obtener la } \\
\text { media) }\end{array}$ & 07. 'COC', 12. 'TRA', 15. 'JUE' & $\begin{array}{l}\text { 02. 'ROP', 07. 'COC', 11. 'CAM', } \\
\text { 12. 'TRA', 14. 'ANI', 17. 'COL' }\end{array}$ \\
\hline $\begin{array}{l}4^{\circ} \text { grupo (ámbitos temáticos } \\
\text { menos productivos) }\end{array}$ & $\begin{array}{l}\text { 03. 'CAS', 04. 'MUE', } \\
\text { 06. 'MES', 09. 'ILU', 13. 'TRC' }\end{array}$ & 06. 'MES', 09. 'ILU', 13. 'TRC' \\
\hline
\end{tabular}

Tabla 5. Clasificación de los centros de interés según la producción de respuestas

Como puede verse (tabla 5), se registra mucha coincidencia en los centros de interés menos productivos, puesto que el 06. 'MES', el o9. 'ILU' y el 13. 'TRC' aparecen como ámbitos muy pobres tanto en nuestra investigación como en las del PPHDL que sirven de contraste. Se observa también que estos estudios coinciden tanto en el campo 16. 'PRO' como en las áreas 07. 'COC' y 12. 'TRA', que aparecen, respectivamente, en el segundo y en el tercer grupo de centros. En cuanto a los estímulos verbales más productivos, se registra de nuevo otra coincidencia en el campo asociativo 05. 'ALI', que figura en las primeras posiciones en esos estudios. Por otro lado, las grandes divergencias se observan, sobre todo, en los ámbitos temáticos 14. 'ANI', 03. 'CAS' y 04. 'MUE'. Los alumnos nativos 
consiguen generalmente los mejores promedios en el centro 14. 'ANI', mientras que en nuestra investigación figura entre los campos que no alcanzan la media. Ello puede ser debido a que este ámbito temático no se ha abordado de manera directa en ninguno de los manuales de ELE que se utilizan en la enseñanza secundaria marroquí. Estos manuales son Español para dialogar (S'houli, Ramdane y Jarif, 2005), Español para progresar (S'houli, Ramdane y Jarif, 2006) y Español para profundizar (S’houli, El Knaiche, Ramdane y Jarif, 2007), dirigidos a los alumnos de Tronco común, $1^{\circ}$ de Bachillerato y $2^{\circ}$ de Bachillerato, respectivamente. ${ }^{8}$ Solo hay unas pocas alusiones al centro 14 . 'ANI' en la unidad didáctica 'Campo y ciudad', que trata, entre otras cosas, algunas actividades laborales relacionadas con la ganadería. Los dos centros siguientes, es decir, el o3. 'CAS' y el 04. 'MUE', pasan del grupo de centros que obtienen la media en nuestro trabajo al último subconjunto en los estudios del PPHDL.

En síntesis, podemos afirmar que la posición rezagada que ocupan algunos campos asociativos tiene su explicación en la escasa atención que le dedican los manuales escolares. Muchos de estos centros de interés se estudian solo en Tronco común, es decir, en el nivel A1, y no se garantiza una continuación en los niveles posteriores. Las áreas temáticas 02. 'ROP' y 17. 'COL' están programadas, a modo de ejemplo, solo en la segunda y en la tercera unidad didáctica del método de nivel inicial, mientras que el o9. 'ILU' aparece de una manera indirecta en la cuarta unidad. De una forma indirecta también se hace referencia, por este orden, a las áreas temáticas o6. 'MES' y 14. 'ANI' en la quinta unidad del mismo manual y en la tercera unidad (Campo y ciudad) del método de nivel intermedio (A2). Esta situación requiere, sin lugar a dudas, echar mano de los resultados que ofrecen los estudios de léxico disponible en español. Muchos investigadores de esta disciplina (Benítez Pérez, 2009; Bartol Hernández, 2010; Paredes García, 2015, entre otros.) insisten en la necesidad de una planificación adecuada y una buena selección del vocabulario que se enseña en las clases de ELE y no dejarlo al capricho de los elaboradores del material didáctico o de los profesores de esta lengua.

\section{2. Producción de vocablos}

La producción de vocablos es otro parámetro cuantitativo que se toma en consideración en los estudios de vocabulario disponible con el fin de analizar la variedad léxica de los encuestados. En nuestro caso, la cantidad de respuestas diferentes que han producido los alumnos en su diccionario definitivo alcanza un total de 4316 unidades distintas, lo que se traduce en una media de 12.33 por informante y de 253.8 por centro de interés.

En la tabla 6 se ofrecen los datos relativos a la producción de palabras distintas y el rango que ocupa cada área temática. 


\begin{tabular}{|c|c|c|c|}
\hline $\mathrm{CI}$ & Rango & Vocablos & Distribución porcentual \\
\hline 01. CUE & 08 & 240 & $5.56 \%$ \\
\hline 02. ROP & 15 & 142 & $3.29 \%$ \\
\hline 03. CAS & 14 & 180 & $4.17 \%$ \\
\hline 04. MUE & 09 & 228 & $5.28 \%$ \\
\hline 05. ALI & 07 & 249 & $5.76 \%$ \\
\hline 06. MES & 16 & 112 & $2.59 \%$ \\
\hline 07. $\mathrm{COC}$ & 10 & 225 & $5.21 \%$ \\
\hline 08. ESC & 03 & 364 & $8.43 \%$ \\
\hline 09. ILU & 13 & 194 & $4.49 \%$ \\
\hline 10. CIU & 01 & 500 & $11.58 \%$ \\
\hline 11. CAM & 02 & 437 & $10.12 \%$ \\
\hline 12. TRA & 11 & 219 & $5.07 \%$ \\
\hline 13. TRC & 05 & 318 & $7.36 \%$ \\
\hline 14. ANI & 12 & 214 & $4.95 \%$ \\
\hline 15. JUE & 06 & 285 & $6.60 \%$ \\
\hline 16. PRO & 04 & 347 & $8.03 \%$ \\
\hline 17. $\mathrm{COL}$ & 17 & 62 & $1.43 \%$ \\
\hline Total & & 4316 & $100 \%$ \\
\hline
\end{tabular}

Tabla 6. Producción de vocablos

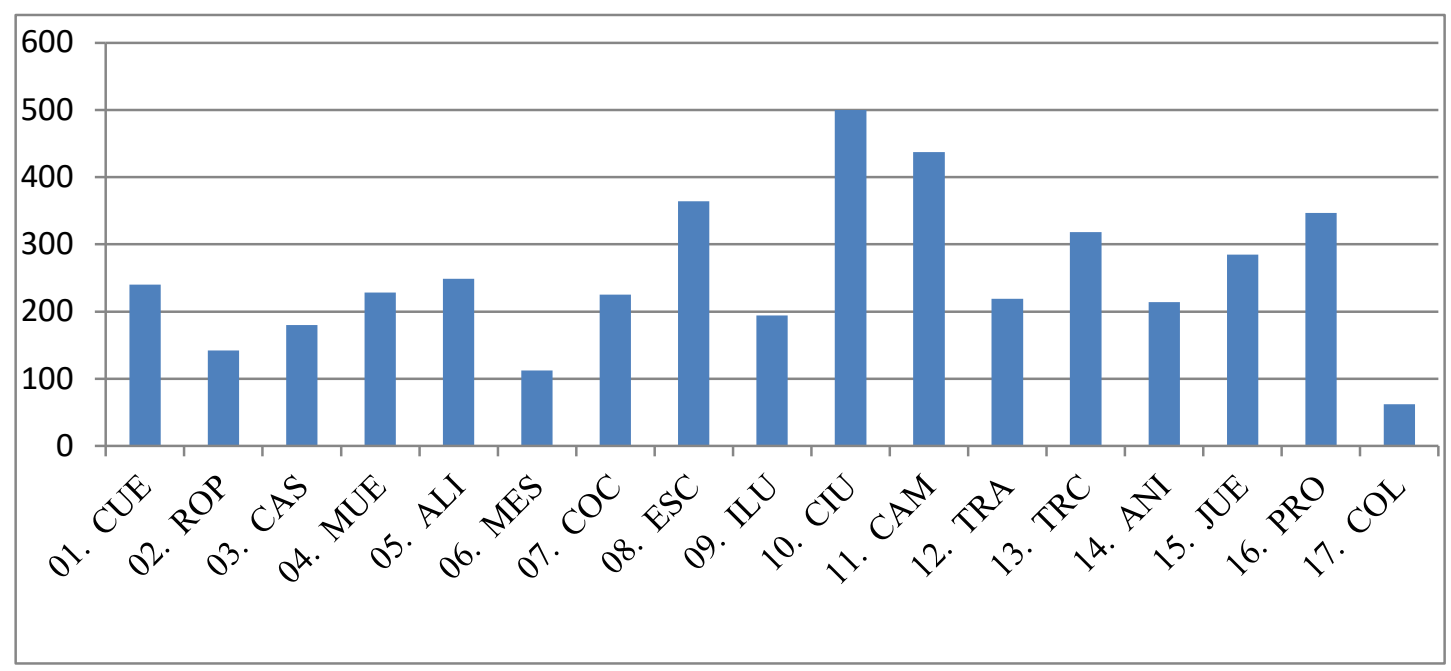

Gráfico 1. Producción de vocablos 
Los datos expuestos en la tabla 6 permiten observar que los alumnos han aportado el mayor número de vocablos en los centros de interés 10. 'CIU' (500 respuestas) y 11. 'CAM' (437 unidades). En la tercera y en la cuarta posición figuran los campos asociativos o8. 'ESC' y 16. 'PRO' con 364 y 347 vocablos, respectivamente. Se trata de unos ámbitos temáticos muy abiertos y que ofrecen al alumnado la posibilidad de mencionar en sus hojas de respuestas muchas asociaciones secundarias y unidades léxicas que pertenecen a diferentes subcampos. Además, estos tres centros forman parte de las áreas más productivas en las investigaciones de léxico disponible dentro del PPHDL. Por otro lado, la menor producción de palabras distintas se registra en el ámbito temático 17. 'COL' con la actualización de solamente 62 vocablos, seguido de 06. 'MES' (112 unidades diferentes). Estos campos asociativos suelen ser generalmente los más pobres en las investigaciones de léxico disponible, puesto que no ofrecen grandes márgenes para aportar muchas asociaciones, y los alumnos se limitan, por lo tanto, a producir un vocabulario muy concreto relacionado estrechamente con estos temas.

Por su parte, las columnas del gráfico 1 reflejan claramente la cantidad desigual de palabras diferentes actualizadas en cada ámbito temático. Se observa también que los encuestados han superado la media de palabras por centro (253.8) solo en seis campos asociativos: 10. 'CIU', 11. 'CAM', 08. 'ESC', 16. 'PRO', 13. 'TRC' y 15. 'JUE', mientras que las áreas 01. 'CUE', 04. 'MUE', 05. 'ALI', 07. 'COC', 12. 'TRA' y 14. 'ANI' no están tampoco muy lejos de alcanzar dicha media. Por último, en el resto de los campos se ha incluido una cantidad menor de respuestas distintas, la cual oscila entre 100 y 200 unidades, excepto en el 17. 'COL', que destaca por su escasa variedad léxica (62 vocablos).

Para terminar esta exposición de los resultados generales, consideramos de gran importancia realizar, a continuación, una comparación cuantitativa entre la producción léxica de los informantes según los totales de palabras y de vocablos. Es sabido que el hecho de actualizar mayor número de repuestas no significa forzosamente aportar mayor cantidad de vocablos (Samper Hernández, 2002, 60). El mejor ejemplo en nuestro caso lo representan los ámbitos temáticos 13. 'TRC', 11. 'CAM' y 17. 'COL', que ocupan, por este orden, las posiciones 15, 10 y 09 según el total de palabras, mientras que según la cantidad de vocablos aparecen en los rangos 05, 02 y 17, respectivamente.

En el gráfico 2 se plasma la cantidad de respuestas producidas según ambos parámetros cuantitativos: la producción de palabras y de vocablos. Como se puede observar, las columnas de este gráfico dejan ver claras divergencias en la producción léxica de los alumnos marroquíes. Su aportación de vocabulario disponible ha variado mucho según el centro de interés y el parámetro cuantitativo contemplado. A este respecto, el rango, por su parte, corrobora estas discrepancias, ya que no existen coincidencias en la posición que ocupan los campos asociativos, excepto el centro 16. 'PRO' que figura en la cuarta posición según ambos criterios. Este campo ocupa este mismo rango en los estudios de léxico dentro del PPHDL según el promedio general de vocablos, mientras que, según el promedio de respuestas, aparece también en este mismo 
puesto $\left(4^{\circ}\right)$ en los estudios realizados en Puerto Rico, Madrid, Ávila, Asturias, Salamanca y Zamora (vid. Samper Padilla et al., 2003, 58).

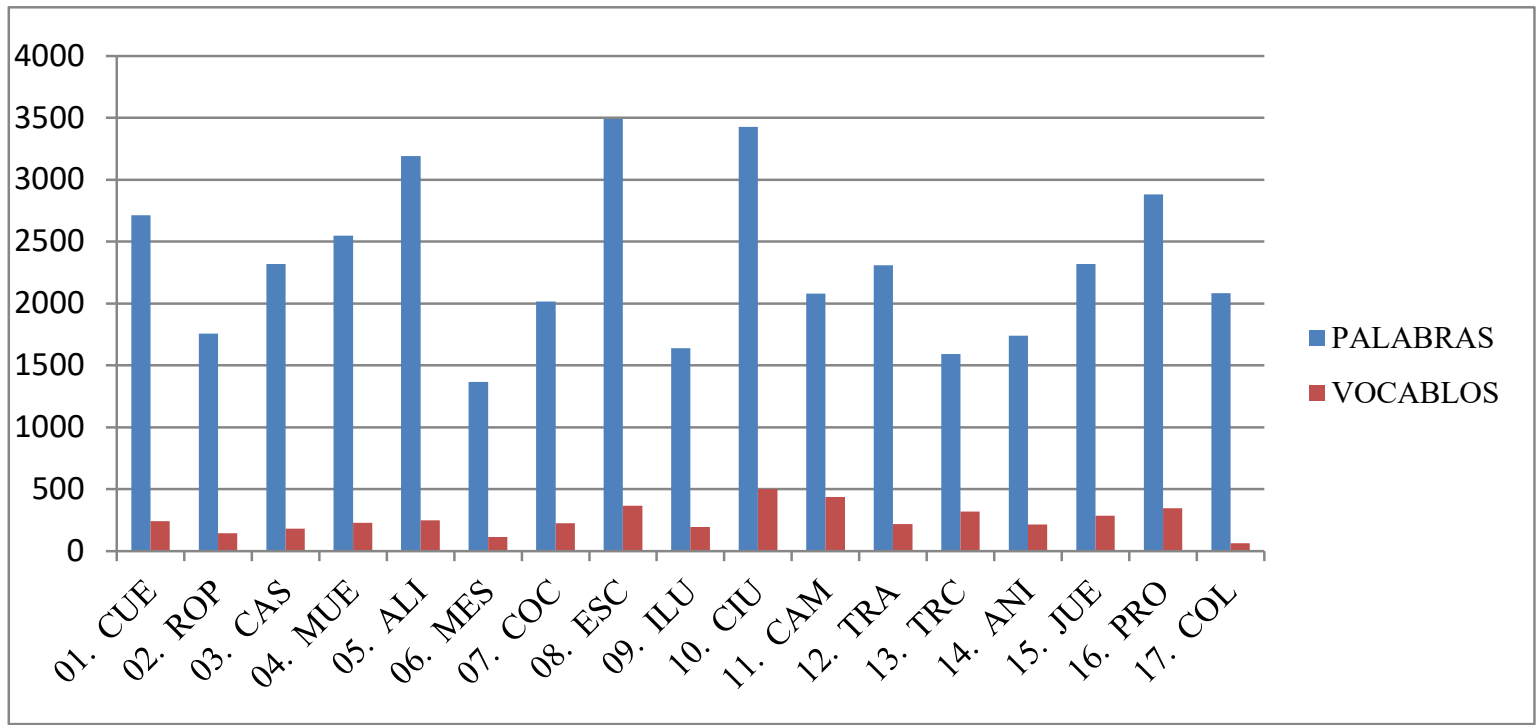

Gráfico 2. Producción de vocablos y de palabras

Las mayores distancias se registran en el rango que concede, por este orden, la producción de palabras y de vocablos a los ámbitos temáticos siguientes: el 13. 'TRC' pasa del $16^{\circ}$ al $5^{\circ}$ puesto, el 17 . 'COL' retrocede del $10^{\circ}$ al $17^{\circ}$ y el 11. 'CAM' mejora su posición ascendiendo de la $10^{\mathrm{a}}$ a la $2^{\mathrm{a}}$. Estos cambios en la posición que ocupan algunos centros se han observado también en muchas investigaciones dentro del PPHDL (López González, 2014, 74): el 13. 'TRC' ha pasado del puesto $16^{\circ}$ según el promedio de palabras al $5^{\circ}$ rango según el promedio general de vocablos, mientras que el 11. 'CAM' ha mejorado su posición pasando de la $8^{\mathrm{a}}$ a la $1^{\mathrm{a}}$ según ambos parámetros cuantitativos, respectivamente.

\section{3. Índice de cohesión}

El índice de cohesión constituye el tercer parámetro cuantitativo que se emplea para analizar la producción léxica de los alumnos. Es un criterio que permite distinguir entre los campos asociativos compactos (cerrados) y los centros difusos (abiertos). Estos valores, que miden el grado de coincidencias y divergencias en cada ámbito, se obtienen dividiendo el promedio de respuestas por el número de palabras diferentes producidas (Echeverría, 1991, 62). Este parámetro permite, por otra parte, evitar la influencia que puede tener la presencia de informantes atípicos en la aportación léxica de un grupo determinado, puesto que un centro de interés registra una mayor disponibilidad léxica solo si destaca por la inclusión de un gran número de vocablos y por la consecución de un alto índice de cohesión. 
La tabla 7 ofrece, además de los índices obtenidos, el rango que ocupa cada campo asociativo. Hay que recordar que cuanto más se acerque este índice a 1, más cerrado será el ámbito temático.

\begin{tabular}{|c|c|c|c|c|}
\hline CI & Rango & $\begin{array}{l}\text { Promedio de palabras } \\
\text { por sujeto }\end{array}$ & NPD & I C \\
\hline 01. CUE & 06 & 7.7 & 240 & 0.032 \\
\hline 02. ROP & 04 & 5.0 & 142 & 0.035 \\
\hline 03. CAS & 02 & 6.6 & 180 & 0.036 \\
\hline 04. MUE & 07 & 7.2 & 228 & 0.031 \\
\hline 05. ALI & 02 & 9.1 & 249 & 0.036 \\
\hline 06. MES & 05 & 3.9 & 112 & 0.034 \\
\hline 07. COC & 10 & 5.7 & 225 & 0.025 \\
\hline 08. ESC & 09 & 9.9 & 364 & 0.027 \\
\hline 09. ILU & 11 & 4.6 & 194 & 0.023 \\
\hline 10. CIU & 15 & 9.7 & 500 & 0.019 \\
\hline 11. CAM & 17 & 5.9 & 437 & 0.013 \\
\hline 12. TRA & 08 & 6.5 & 219 & 0.029 \\
\hline 13. TRC & 16 & 4.5 & 318 & 0.014 \\
\hline 14. ANI & 14 & 4.9 & 214 & 0.022 \\
\hline 15. JUE & 11 & 6.6 & 285 & 0.023 \\
\hline 16. PRO & 11 & 8.2 & 347 & 0.023 \\
\hline 17. COL & 01 & 5.9 & 62 & 0.095 \\
\hline
\end{tabular}

Tabla 7. Índice de cohesión de cada centro de interés

Se desprende de esta tabla que el mayor índice de cohesión se registra en el centro de interés 17. 'COL' (0.095), a pesar de que es un ámbito que no ha alcanzado la media según el promedio de respuestas y, además, constituye el más pobre en vocablos. Este índice significa que las respuestas de los encuestados han coincidido mucho en este ámbito, que se sitúa, además, a una larga distancia de los demás campos asociativos. En la segunda posición figuran las áreas temáticas 03. 'CAS' y 05. 'ALI', que han obtenido el mismo índice de cohesión, es decir, o.036 puntos. Estos tres centros constituyen, por lo tanto, los más compactos. Por el contrario, en la posición más rezagada aparece el ámbito 11. 'CAM', puesto que ostenta el índice de cohesión más bajo (o.013). También se registran los menores valores en los campos 13. 'TRC' y 10. 'CIU', cuyos índices no superan 0.014 y 0.019, respectivamente. Estos tres estímulos verbales constituyen, por consiguiente, los más abiertos, es decir, los que contienen mayor variedad de asociaciones léxicas. 
A continuación, en la tabla 8 se clasifican los centros de interés según el índice de cohesión obtenido tanto por los alumnos marroquíes como por los nativos dentro del PPHDL (López González, 2014, 80):

\begin{tabular}{|c|c|c|}
\hline Grupo & $\begin{array}{l}\text { Centros de interés } \\
\text { en el presente estudio }\end{array}$ & $\begin{array}{l}\text { Centros de interés } \\
\text { en el PPHDL }\end{array}$ \\
\hline $1^{\mathrm{er}}$ grupo: centros más compactos & 17. 'COL' & 01. 'CUE' \\
\hline $\begin{array}{l}2^{\circ} \text { grupo: su índice de cohesión } \\
\text { alcanza o supera la media }\end{array}$ & $\begin{array}{l}\text { 01. 'CUE', 02. 'ROP', 03. 'CAS', } \\
\text { 04. 'MUE', 05. 'ALI', 06. 'MES' }\end{array}$ & $\begin{array}{l}\text { 02. 'ROP', 14. 'ANI', 12. 'TRA', } \\
\text { 03. 'CAS', 06. 'MES', 04. 'MUE', } \\
\text { 05. 'ALI', }\end{array}$ \\
\hline $\begin{array}{l}3^{\text {er }} \text { grupo: su índice de cohesión no } \\
\text { alcanza la media }\end{array}$ & $\begin{array}{l}\text { 07. 'COC', 08. 'ESC', 09. 'ILU', } \\
\text { 12. 'TRA', 14. 'ANI', 15. 'JUE', } \\
\text { 16. 'PRO' }\end{array}$ & 07. 'COC', 08. 'ESC', 09. 'ILU' \\
\hline $4^{\circ}$ grupo: centros más difusos & 11. 'CAM', 13. 'TRC', 10. 'CIU' & $\begin{array}{l}\text { 16. 'PRO', 10. 'CIU', 15. 'JUE', } \\
\text { 13. 'TRC', 11. 'CAM' }\end{array}$ \\
\hline
\end{tabular}

Tabla 8. Clasificación de los centros de interés según el índice de cohesión

Como puede verse, el índice de cohesión ofrece generalmente resultados muy similares y convergentes, puesto que los alumnos marroquíes coinciden con sus homólogos nativos en el carácter difuso o compacto de muchos centros de interés. Sin embargo, destacan las divergencias observadas en cinco ámbitos temáticos. En los estudios del PPHDL, que no contemplan generalmente el centro 17. 'COL', el campo asociativo 01. 'CUE' ocupa la primera posición y constituye, por lo tanto, el más cohesionado, mientras que en nuestra investigación aparece en un puesto rezagado $\left(6^{\circ}\right)$. Los ámbitos temáticos 14. 'ANI' y 12. 'TRA' manifiestan también esta divergencia entre ambos grupos, puesto que su índice de cohesión supera la media en los nativos, mientras que en los marroquíes no alcanza dicho valor: en el PPHDL figuran, por este orden, en el $3^{\text {er }}$ y en el $4^{\circ}$ puesto, mientras que en los marroquíes retroceden notablemente al rango $14^{\circ}$ y $8^{\circ}$, respectivamente. Por último, el índice obtenido en los campos léxicos 15. 'JUE' y 16. 'PRO' también deja ver diferencias relevantes en ambas investigaciones, puesto que forman parte del grupo de centros más abiertos en los nativos, mientras que en nuestro estudio aparecen en la tercera casilla de la tabla, es decir, los ámbitos que no alcanzan la media, pero tampoco están muy lejos de obtener este valor.

Para terminar este apartado, consideramos muy interesante comparar también los índices de cohesión obtenidos por los marroquíes con los de otras investigaciones de léxico disponible en ELE. En la tabla 9 exponemos los centros de interés que ocupan las dos primeras posiciones (los más cerrados) y los que figuran en los dos últimos rangos (los más abiertos): 


\begin{tabular}{|l|l|l|l|l|l|}
\hline \multicolumn{1}{|c|}{ Rango } & \multicolumn{1}{|c|}{ Marruecos } & \multicolumn{1}{|c|}{ Finlandia } & \multicolumn{1}{c|}{ China } & \multicolumn{1}{|c|}{ Polonia } & Rumanía \\
\hline Primero & 17. 'COL' & 06. 'MES' & 06. 'MES' & 01. 'CUE' & 17. 'COL' \\
\hline Segundo & $\begin{array}{l}\text { 03. 'CAS', } \\
\text { 05. 'ALI' }\end{array}$ & 12. 'TRA' & 01. 'CUE' & $\begin{array}{l}\text { 02. 'ROP', 03. 'CAS', 14. } \\
\text { 'ANI' }\end{array}$ & 14. 'ANI' \\
\hline \hline Penúltimo & 13. 'TRC' & 07. 'COC' & 15. 'JUE' & 15. 'JUE', 11. 'CAM' & 15. 'JUE' \\
\hline \hline Último & 11. 'CAM' & 13. 'TRC' & 11. 'CAM' & 13. 'TRC' & 13. 'TRC' \\
\hline
\end{tabular}

Tabla 9. Los centros de interés más cohesionados y los más abiertos

Se desprende de esta tabla que los centros 06. 'MES' y 01. 'CUE' que encabezan, por este orden, la lista de los ámbitos más cerrados en las muestras finlandesa, china y polaca, no figuran entre los más compactos en la muestra marroquí. Sin embargo, este último grupo coincide con los rumanos en la consecución de un alto grado de convergencias en las respuestas incluidas en el área temática 17. 'COL'. Se observa, por otra parte, que los campos léxicos más abiertos en los marroquíes, es decir, el 11. 'CAM' y 13. 'TRC', aparecen también entre los más difusos en las demás investigaciones. Coinciden totalmente con los chinos en el estímulo verbal que ocupa la última posición (11. 'CAM'), mientras que el 13. 'TRC' pasa del penúltimo rango en Marruecos al último puesto en Finlandia, Polonia y Rumanía.

\section{CONCLUSIONES}

El estudio de léxico disponible en español de los alumnos marroquíes de enseñanza media tiene como objetivo principal presentar los resultados obtenidos desde el punto de vista cuantitativo y contrastarlos con los de otros estudios de vocabulario español de esta misma índole. El análisis de la producción tanto de palabras como de vocablos en los diferentes centros de interés considerados permite llegar a las siguientes conclusiones parciales:

1. La cantidad de palabras producidas por los encuestados marroquíes es de 39462 unidades, mientras que el número de vocablos solo ha alcanzado 4316 respuestas diferentes. Estos totales se pueden traducir en una media de 2321.3 respuestas, frente a 253.8 vocablos según el segundo parámetro.

2. Los ámbitos temáticos más productivos según la cantidad de palabras son o8. 'ESC', 10. 'CIU' y 05.'ALI', frente a los centros 10. 'CIU', 11. 'CAM', o8. 'ESC' y 16. 'PRO', que ocupan las primeras posiciones según el número de vocablos. Por otro lado, los campos asociativos más pobres según el primer criterio son 06. 'MES', 13. 'TRC' y 
09. 'ILU', frente a los centros 17. 'COL', 06. 'MES' y 02. 'ROP', que aparecen en las últimas posiciones según la producción de respuestas diferentes.

3. Las listas de los centros que ocupan las primeras y las últimas posiciones según la producción de palabras dejan ver muchas coincidencias al comparar nuestros resultados con los de otras investigaciones de esta misma índole. No obstante, resulta curioso el caso del campo 08. 'ESC', que no aparece en el listado de los ámbitos más ricos en los otros estudios de vocabulario disponible en ELE, mientras que en los marroquíes constituye el más productivo. Llama la atención, por otro lado, el caso de las áreas temáticas 02. 'ROP' y 14. 'ANI', que ocupan posiciones muy rezagadas en Marruecos, pero no figuran en las listas de los cinco últimos centros más pobres en las demás investigaciones.

4. Según el índice de cohesión, el campo asociativo 17. 'COL' (0.095) es el más compacto. Esto significa que las respuestas de los encuestados han coincidido mucho en este centro. A una larga distancia se sitúan los ámbitos 03. 'CAS' y 05. 'ALI', que ocupan la segunda y la tercera posición. Por el contrario, los campos 11. 'CAM', 13. 'TRC' y 10. 'CIU' manifiestan una gran diversificación léxica y constituyen, por lo tanto, las áreas temáticas más abiertas, puesto que ofrecen a los informantes la posibilidad de incluir respuestas que pertenecen a diferentes categorías léxicas. El carácter difuso o compacto de los estímulos verbales propicia muchas coincidencias entre nuestros resultados y los que proporcionan los estudios de vocabulario disponible dentro del PPHDL.

5. La posición rezagada de algunos centros de interés según la producción léxica se debe quizá a la escasa atención que reciben en los manuales escolares, ya que muchos temas se estudian solo en el nivel inicial, y luego no se garantiza una continuación en los niveles posteriores. Esta pobreza léxica cuestiona la metodología y los criterios que emplean los especialistas y elaboradores del material didáctico a la hora de seleccionar y planificar el vocabulario español que se quiere explotar en las aulas.

En definitiva, se trata de unas conclusiones meramente cuantitativas que serán enriquecidas y completadas con otros análisis cualitativos más profundos para descubrir las tendencias y demás características del léxico disponible en español de los alumnos marroquíes y compararlas con los resultados de otras investigaciones de la misma índole. Ofrecen también otras vías de investigación científica sobre la selección del vocabulario explotado y su planificación curricular con el fin de mejorar la enseñanza-aprendizaje de la lengua española en Marruecos. Los encargados de elaborar el material didáctico en este país están llamados a aprovechar las diferentes posibilidades y ventajas que aporta la disponibilidad léxica a la didáctica de esta lengua. Este tipo de estudios se basa en una metodología rigurosa capaz de garantizar una buena selección del vocabulario y su gradación según el nivel educativo. 


\section{NOTAS}

$1 \quad$ Para una información más detallada sobre los estudios de léxico disponible en ELE véanse Hidalgo Gallardo (2017) y Aabidi (2019).

$2 \quad$ Sobre el Proyecto Panhispánico de Disponibilidad Léxica pueden consultarse los trabajos de Samper Padilla et al. (2003) y Samper Padilla y Samper Hernández (2006) o la página electrónica de este proyecto: http://www.dispolex.com.

$3 \quad$ Desgraciadamente, en la Academia Regional de Guelmim-Oued Noun no hemos podido contar con alumnos que estudian ELE.

$4 \quad$ La estratificación de la muestra según el nivel sociocultural de los informantes se ha basado en los dos parámetros - nivel de estudios de los padres y su profesión- empleados por Samper Padilla y Hernández Cabrera (1997, 230-233).

5 Los centros de interés en las investigaciones del PPHDL están ordenados según la media general de respuestas por informante (Samper Padilla y Samper Hernández, 2006, 31).

6 En las dos primeras casillas que corresponden al rango, aparecen dos números porque hay investigaciones que contemplan 17 centros y otras que analizan solo los 16 campos tradicionales.

7 Aunque nos basamos en la clasificación de los citados autores, en nuestro estudio hemos unificado el cuarto y el quinto grupo (los centros que nunca alcanzan la media y los menos productivos, respectivamente) en una sola casilla.

8 Aunque estos manuales son los más usados en la mayoría de los institutos de enseñanza secundaria en Marruecos, los profesores de ELE pueden utilizar también cualquier otro soporte pedagógico siempre que se respeten los contenidos temáticos, funcionales, morfosintácticos y socioculturales programados para cada nivel y que vienen en las orientaciones pedagógicas del Ministerio de Educación Nacional.

\section{REFERENCIAS BIBLIOGRÁFICAS}

AABIDI, L. 2019. "La disponibilidad léxica en español como lengua extranjera: dos décadas de investigación científica”, MarcoELE, 28.

BARTOL HERNÁNDEZ, J. A. 2010. "Disponibilidad léxica y selección del vocabulario", en Castañer Martín, R. M. y Lagüéns Gracia, V. (eds.), De moneda nunca usada: Estudios dedicados a José M. ${ }^{a}$ Enguita Utrilla. Zaragoza: Institución Fernando el Católico, pp. 85-107.

BENÍTEZ PÉREZ, P. 2009. “¿Qué vocabulario hay que enseñar en las clases de español como lengua extranjera?”, MarcoELE, 8.

BENYAYA, Z. 2007. "La enseñanza del español en Marruecos: del pasado al presente", Porta Linguarum, 7, pp. 167-180.

CARCEDO GONZÁLEZ, A. 1997-1998. "Desarrollo de la competencia léxica en español LE: análisis de cuatro fases de disponibilidad”, Pragmalingüística, 5-6, pp. 75-94. 
CARCEDO GONZÁLEZ, A. 200o. Disponibilidad léxica en español como lengua extranjera: el caso finlandés (estudio del nivel preuniversitario y cotejo con tres fases de adquisición). Turku: Turun Yliopiston Julkaisuja.

CHARIA, Z. 2014. "El hispanismo marroquí y el protectorado español en Marruecos", Hispanista, XV(57).

ECHEVERRÍA, M. S. 1991. "Crecimiento de la disponibilidad léxica en estudiantes chilenos de nivel básico y medio”, en López Morales, H. (ed.), La enseñanza del español como lengua materna. Río Piedras: Editorial de la Universidad de Puerto Rico, pp. 61-78.

GALLEGO GALLEGO, D. J. 2014. "Léxico disponible de 82 inmigrantes estudiantes de español en la ciudad de Alcalá de Henares”, Lengua y migración, 6(2), pp. 95-123.

HIDALGO GALLARDO, M. 2017. "Sobre la disponibilidad léxica en ELE. Revisión de la literatura”, Boletín ASELE, 56, pp. 83-94.

INSTITUTO CERVANTES. 2007. Plan curricular del Instituto Cervantes. Niveles de referencia para el español. Disponible en: https://cvc.cervantes.es/ensenanza/biblioteca ele/plan_curricular/

JIMÉNEZ BERRIO, F. 2013. Léxico disponible de inmigrantes escolares no hispanohablantes. Pamplona: Servicio de Publicaciones de la Universidad de Navarra.

LIN, J. 2012. "El estudio de disponibilidad léxica de los estudiantes chinos de español como lengua extranjera”, MarcoELE, 14.

LÓPEZ GONZÁLEZ, A. M. 2010. "La evaluación del desarrollo de la competencia léxica en L2 por medio de la disponibilidad léxica”, redELE, 18.

LÓPEZ GONZÁLEZ, A. M. 2014. Disponibilidad léxica. Teoría, método y análisis. Lodz: Wydawnictwo Uniwersytetu Łódzkiego.

MENDOZA PUERTAS, J. D. 2018. "El léxico disponible de 82 estudiantes coreanos de español como lengua extranjera”, MarcoELE, 26.

PAREDES GARCÍA, F. 2015. "Disponibilidad Léxica y enseñanza de ELE: el léxico disponible como fuente curricular y como recurso en el aula”, LinRed, XIII.

SAMPER HERNÁNDEZ, M. 2002. Disponibilidad léxica en alumnos de español como lengua extranjera. Málaga: ASELE.

SAMPER PADILLA, J. A. 1998. "Criterios de edición del léxico disponible: sugerencias", Lingüística, 10, pp. 311-333.

SAMPER PADILLA, J. A., BELLÓN FERNÁNDEZ, J. J. y SAMPER HERNÁNDEZ, M. 2003. "El proyecto de estudio de la disponibilidad léxica en español”, en Wotjak, G. (coord.), Pautas y pistas en el análisis del léxico hispano(americano). Madrid: IberoaméricaVervuert, pp. 27-140.

SAMPER PADILLA, J. A. y HERNÁNDEZ CABRERA, C. E. 1997. "El estudio de la disponibilidad léxica en Gran Canaria: datos iniciales y variación sociolingüística”, en Almeida, M. y Dorta, J. (eds.), Contribuciones al estudio de la lingüística hispánica. Homenaje al profesor Ramón Trujillo. Tomo II. Barcelona: Montesinos, pp. 229-239.

SAMPER PADILLA, J. A. y SAMPER HERNÁNDEZ, M. 2006. "Aportaciones recientes de los estudios de disponibilidad léxica”, LynX: Panorámica de Estudios lingüísticos, 5, pp. 5-95. 
SANDU, B. M. 2014. Léxico disponible de alumnos rumanos que aprenden español como lengua extranjera en centros escolares bilingües rumano-españoles. Tesis doctoral. Las Palmas de Gran Canaria: Universidad de Las Palmas de Gran Canaria.

SERFATI, M. y AABIDI, L. 2013. Disponibilidad léxica de ELE en Marruecos (Niveles de Secundaria y Enseñanza Superior en la región Souss Massa Drâa). Agadir: Universidad Ibn Zohr.

S’HOULI, A., EL KNAICHI, B., RAMDANE, F. y JARIF, S. 2007. Español para profundizar. Rabat: El Maarif al Jadida.

S'HOULI, A., RAMDANE, F. y JARIF, S. 2005. Español para dialogar. Rabat: El Maarif al Jadida.

S'HOULI, A., RAMDANE, F. y JARIF, S. 2006. Español para progresar. Rabat: El Maarif al Jadida.

\section{NOTA SOBRE ELAUTOR}

Lahoussine Aabidi es profesor titular de ELE en la enseñanza secundaria cualificante en Marruecos. Es también miembro del grupo de investigación EDOCSUR de la Universidad Ibn Zohr (Agadir). En la actualidad prepara su tesis doctoral en la Universidad de Las Palmas de Gran Canaria, España. 